\title{
USING TRUCKSIM FOR A VIRTUAL LONGITUDINAL SLOPE TEST PROCEDURE
}

\author{
Petru ROȘCA \\ Military Equipment and Technologies Research Agency, Bucharest, Romania \\ rosca_petru2000@yahoo.com \\ Gheorghe OLARU \\ Military Technical Academy "Ferdinand I", Bucharest, Romania \\ olaru56@yahoo.com \\ Dorel BADEA \\ “Nicolae Bălcescu” Land Forces Academy, Sibiu, Romania \\ dorel.badea@yahoo.com
}

\begin{abstract}
This paper presents a methodology to validate a virtual test procedure for vehicle gradeability performance. It is a test dedicated to both the civilian and military off-road vehicles, an $8 \times 8$ Armoured Personnel Carrier being selected for the purpose of the paper. TruckSim is the software used to implement the vehicle math model and the test procedure. The simulation results are compared with the experimental results for a $60 \%$ gradient. The vehicle speed and the engine speed graphics, the statistical analyze, and the comparison between the simulation test animation and the experimental test movie are the methods applied to validate the virtual longitudinal slope test procedure.
\end{abstract}

KEYWORDS: TruckSim, vehicle, simulation, test, slope

\section{Introduction}

The military vehicles are recognizable by the off-road performances they have to perform, among which being the capability for operating on the $60 \%$ longitudinal gradient. In order to evaluate wheeled vehicles performance, the test is performed under specific procedures (NATO, 1992) or (TOP 2-2-610, 2009). However, prior to the type approval activity, theoretical calculations are required in order to establish a vehicle configuration that fulfills the required performance criterion. Also, through the vehicle design project, speed limits can be imposed for slopes smaller than $60 \%$. Thus, (TOP 2-2-610, 2009) recommends the determination of vehicle speed on longitudinal slopes with variable degrees of grade $-2 \%, 3 \%, 5 \%, 10 \%, 15 \%, 20 \%$, $30 \%, 40 \%$, and $50 \%$. It is extremely difficult to identify ramps of all categories that ensure constant longitudinal gradient over a roadway length long enough to accelerate, to reach and to maintain the corresponding maximum speed. There is the option of indirect determination by means of the drawbar pull performance (TOP 2-2-610, 2009), still a constricting test procedure because of the difficulty to assure safety conditions for the tested vehicle and towed dynamometer tandem at high speeds. At the same time, the climatic conditions may delay the test accomplishment - the grades must be dry, clean and provide good road adherence, which means restrictions during winter or rainy weather. From these reasons, it is 
necessary to develop and validate a virtual test procedure to certify the longitudinal grade performance of a military vehicle. This method is also accepted by European legislation (Directive 2007/46/EC) in order to demonstrate the capability of off-road vehicles to be able to negotiate a 25-percent grade. The paper proposes to accomplish this objective by using the TruckSim software developed by Mechanical Simulation. The virtual test procedure is validated with the results of a real test on a paved slope.

\section{Vehicle and Procedure Configuration}

The vehicle used for the purpose of this paper is Saur2, an $8 \times 8$ Armoured Personnel Carrier (APC) produced by U.A. Moreni (Figure no. 1).

In (Saur 2 Technical paper, 2017) are presented the main characteristics:

crew: 11, including the driver; engine: Diesel, Euro 3, 240 kW; gearbox: automated;

transfer case: 2 speed; transmission: $8 \times 8$ or $4 \times 4$ (rear); independent suspension; tire: $14.00 \times 20$; gross vehicle weight: $16000 \mathrm{~kg}$.

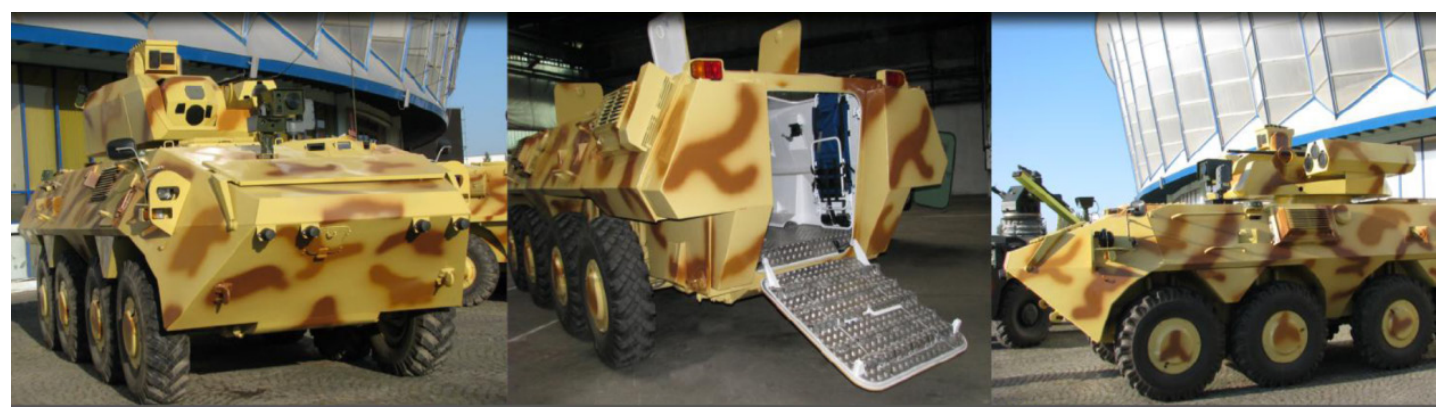

Figure no. 1: SAUR 2

(Source: Saur 2 Technical paper, 2017)

All these characteristics are used to including also the module for slope configure the math model of Saur 2 in procedure, the controls for running the math TruckSim. The open window of the model, and the access to the plots and to the application is presented in Figure no. 2, video for visualizing simulation results.

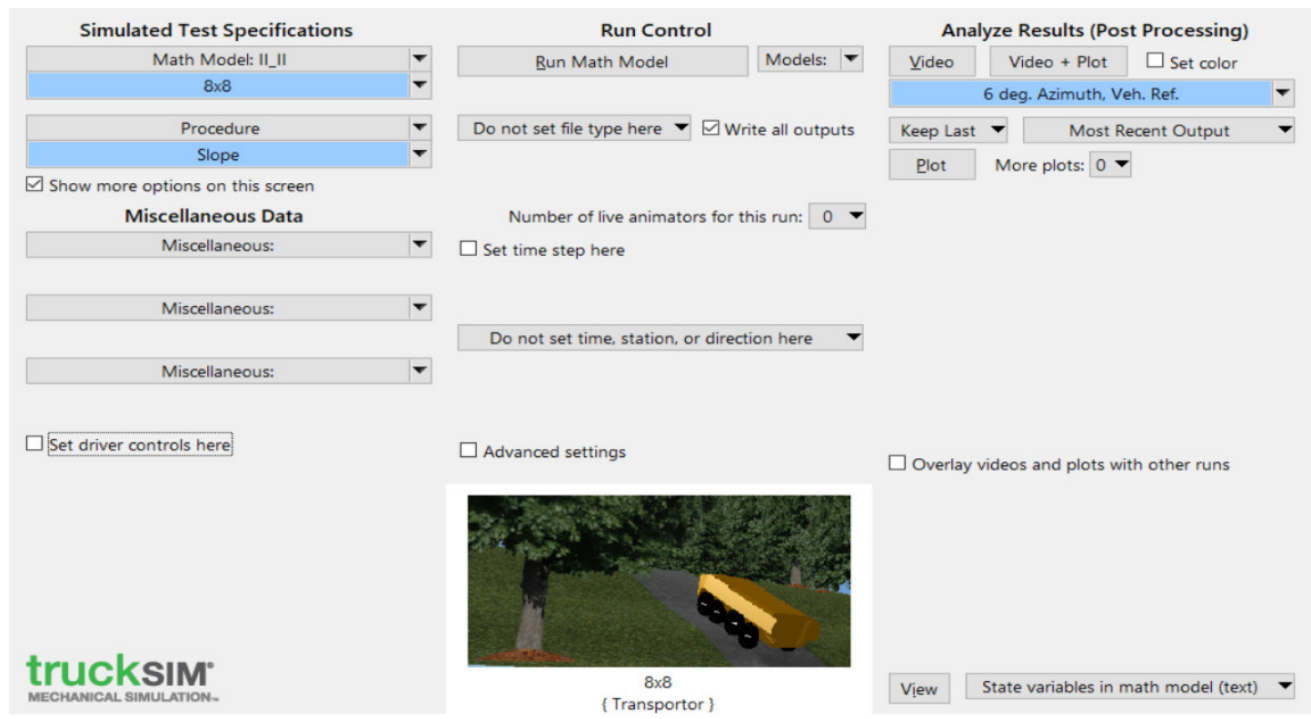

Figure no. 2: TruckSim open window for slope test procedure 
For the slope procedure, the configuration includes the full throttle position, first gear, no direction offset, and no braking. The real gradient is $60 \%$, but the track is characterized by some small road waves which are also implemented in TruckSim. This software allows to import GPS data, so the path is better defined.

\section{Experimental and Simulation Results}

For experimental test the vehicle was instrumented with the following equipment:

VBOX 3i $100 \mathrm{~Hz}$ GPS Data Logger from Racelogic - used for speed, distance, time, latitude and longitude coordinates, and relative height measurements;
HHT20-ROS Remote Optical Sensor from OMEGA Technologies Company used for engine speed measurements.

For the virtual test the vehicle is placed at the base of the slope and the simulation is run till the end of the slope.

Both types of results are extracted from the vehicle running along the slope, since the beginning of the two tests are not identical. Figure no. 1 illustrates the speed, while the Figure no. 2 presents the engine speed. It can be noticed that the vehicle speed increases at the end of the test due to the fact that the gradient almost finishes and the path tends to become horizontal. The oscillations of the vehicle speed for both tests, and especially for simulation, are normal and reflect the road waves.

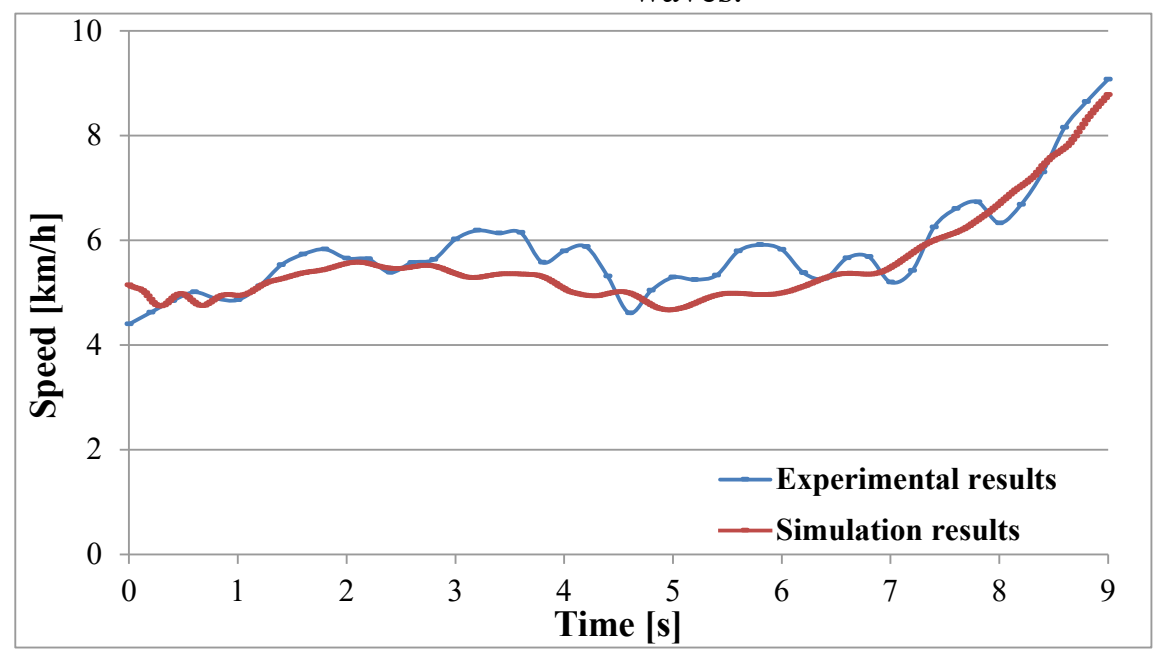

Figure no. 3: Vehicle speed

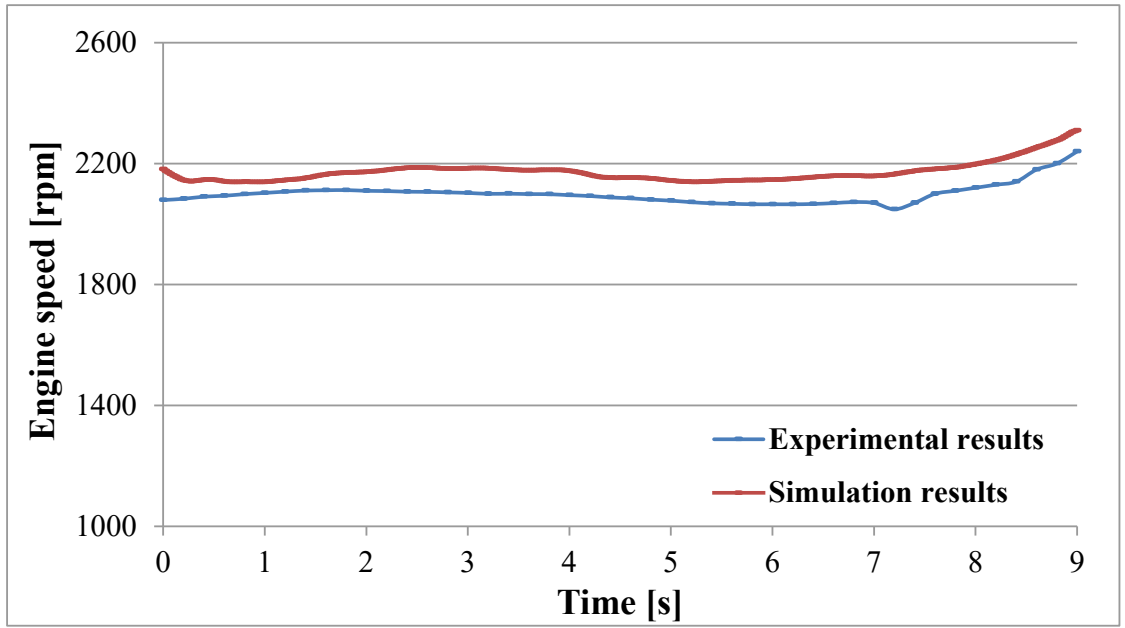

Figure no. 4: Engine speed 
The facilities existing in TruckSim are used for this test procedure to create a virtual world. It is designed and imported an $8 \times 8$ APC hull to configure a closed shape virtual vehicle. Moreover, the trees are placed along the path in order to reflect the reality. Not so important for the purpose of this paper, the
Figure no. 5 emphasizes the possibility to implement the test procedure in the capability of a simulator. The military drivers should be familiarized with the high gradients all the more so as from there only the sky can be seen. Consequently, the first psychological impact is tough and unpleasant.

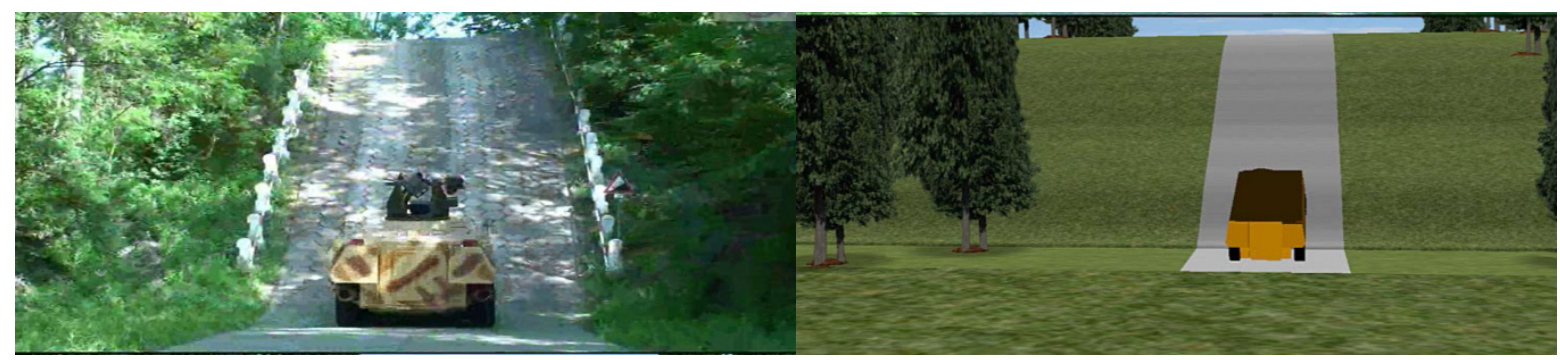

Figure no. 5: Real test and simulation movies comparison

\section{Experimental and Simulation Results Comparison}

Since the Figure no. 3 and Figure no. 4 offer only a subjective visual comparison, an objective statistical one is necessary.

That's why, in line with the work of Hossam Ragheb (2014) the following statistical criteria are used:

- the root mean square (RMS) for simulation results should agree with the RMS for experimental results within $\pm 10 \%$;

- the Skewness value for simulation results should agree with the Skewness for experimental results within $\pm 50 \%$;

- the Kurtosis value for simulation results should agree with the Kurtosis for experimental results within $\pm 50 \%$.

Also, Robinson, Rais-Rohani and Mason (2016) have compared the predicted versus measured values by representing them in graphics. Overmore, the normalized root mean squared error (NRMSE) has been calculated - a lower value meaning a better correlation between the two types of results.

\subsection{Simulation Vehicle Speed Versus Experimental Vehicle Speed}

The statistical values for the vehicle speed are presented in Table no. 1 . Together with an $8.20 \%$ NRMSE, all these values indicate a proper correlation.

Table no. 1

Vehicle speed statistical values

\begin{tabular}{|c|c|c|c|c|c|}
\hline $\begin{array}{c}\text { Validation } \\
\text { coefficient }\end{array}$ & $\begin{array}{c}\text { Experimental } \\
\text { results }\end{array}$ & $\begin{array}{c}\text { Simulation } \\
\text { results }\end{array}$ & $\begin{array}{c}\text { Relative } \\
\text { difference }\end{array}$ & $\begin{array}{c}\text { Validation } \\
\text { criteria }\end{array}$ & $\begin{array}{c}\text { Validation } \\
\text { decision }\end{array}$ \\
\hline RMS & 5.88 & 5.63 & $5.22 \%$ & $\pm 10 \%$ & Yes \\
\hline Skewness & 1.66 & 1.95 & $-18.97 \%$ & $\pm 50 \%$ & Yes \\
\hline Kurtosis & 6.13 & 6.30 & $-2.73 \%$ & $\pm 50 \%$ & Yes \\
\hline
\end{tabular}

Figure no. 6 relates the experimental versus simulation results for vehicle speed. Delimitation lines are marked to group the data points falling within the sectors associated. Almost $74 \%$ of the data points are below the medium line $Y=X$, which means that the virtual longitudinal slope test procedure slightly underestimate the values of the vehicle speed. 


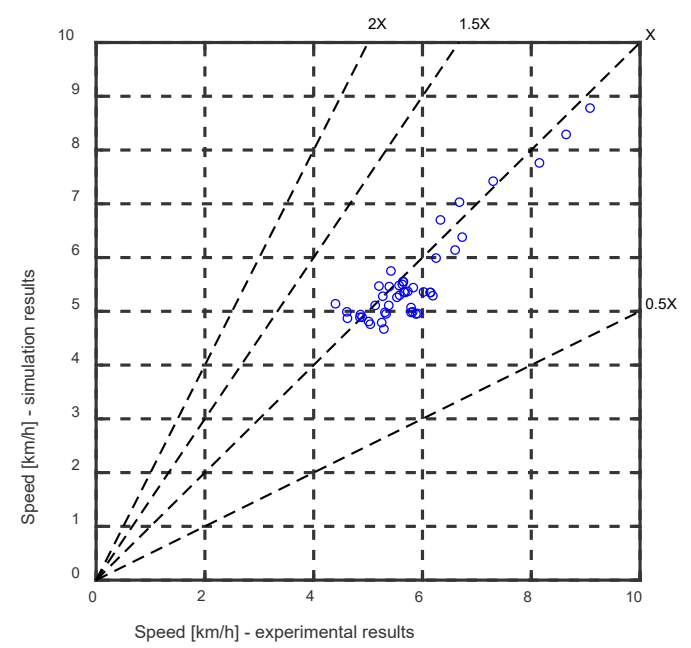

Figure no. 6: Experimental versus simulation vehicle speed

\subsection{Simulation Engine Speed Versus} Experimental Engine Speed

The statistical values for the engine speed are presented in Table no. 2.
Together with a $3.44 \%$ NRMSE, all these values indicate a proper correlation.

Table no. 2

Engine speed statistical values

\begin{tabular}{|c|c|c|c|c|c|}
\hline $\begin{array}{c}\text { Validation } \\
\text { coefficient }\end{array}$ & $\begin{array}{c}\text { Experimental } \\
\text { results }\end{array}$ & $\begin{array}{c}\text { Simulation } \\
\text { results }\end{array}$ & $\begin{array}{c}\text { Relative } \\
\text { difference }\end{array}$ & $\begin{array}{c}\text { Validation } \\
\text { criteria }\end{array}$ & $\begin{array}{c}\text { Validation } \\
\text { decision }\end{array}$ \\
\hline RMS & 2099.66 & 2172.61 & $-3.47 \%$ & $\pm 10 \%$ & Yes \\
\hline Skewness & 2.04 & 2.03 & $0.59 \%$ & $\pm 50 \%$ & Yes \\
\hline Kurtosis & 8.31 & 7.57 & $8.85 \%$ & $\pm 50 \%$ & Yes \\
\hline
\end{tabular}

Figure no. 7 relates the experimental versus simulation results for the engine speed. Delimitation lines are marked to group the data points falling within the above the medium line $\mathrm{Y}=\mathrm{X}$, which means that the virtual longitudinal slope test procedure overestimate the values of the engine speed. sectors associated. All the data points are

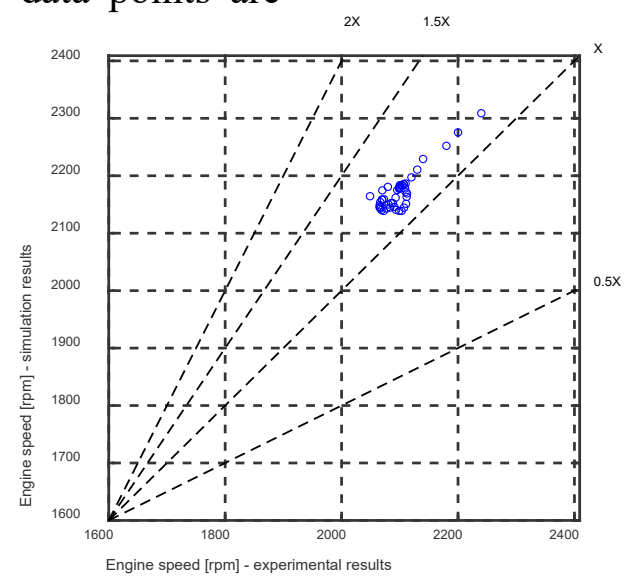

Figure no. 7: Experimental versus simulation engine speed 


\section{Conclusions}

This paper responds to an actual demand of the technical field to transpose not only constructive - functional elements, but also the testing - evaluation procedures into the virtual environment. The exposed method may be a model for all the procedures related to the type approval of both the military and the civilian vehicles. TruckSim software has been selected to define the virtual procedure because of its key-benefits for such objective - intuitive interface, simplicity of the model,
3D animation, real-time simulation. The designated vehicle - Saur 2 APC was tested both in the real and the virtual environments. The results were overlay to have a first visual impact assessment. The statistical analyze confirms the validation of the virtual model by means of associated coefficients. This gives confidence to estimate performance of Saur 2 in other conditions than the exposed one, and also encourages the use of the test procedure for other types of vehicles.

\section{REFERENCES}

European Parliament (2007). Framework Directive 2007/46/EC. establishing a framework for the approval of motor vehicles and their trailers, and of systems, components and separate technical units intended for such vehicles. Brussels, Belgium.

North Atlantic Treaty Organization (NATO). (1992). AVTP 03-90 Gradients and side-slopes, Military Agency for Standardization. Brussels, Belgium.

Ragheb, H. (2014). Torque Control Strategy for Off-Road Vehicle Mobility. PhD Thesis. Ontario, Canada: University of Ontario Institute of Technology.

Robinson, J. et. al. (2016). Multi-objective traction optimization of vehicles in loose dry sand using the generalized reduced gradient method. Journal of Terramechanics, doi:10.1016/j.jterra.2015.10.002

The United States Army, Aberdeen Test Center (2009). TOP 2-2-610 Gradeability and side slope performance. Aberdeen, Maryland.

Uzina Automecanica Moreni. (2017). Saur 2 Tehnical paper. available at: http://www.uamoreni.ro/fise/Saur2RO.pdf, accessed on 24 September 2017. 